\title{
The clinical significance of cytokeratin 20 staining pattern in Merkel cell carcinoma
}

\author{
Yasuhiro Sakata $^{1}$, Yutaka Inaba,*, Kayo Kunimoto ${ }^{2}$, Chikako Kaminaka ${ }^{2}$, Yuki Yamamoto ${ }^{2}$, \\ Yoshifumi Iwahashi ${ }^{3}$, Shin-ichi Murata ${ }^{3}$, Shinichi Asamura ${ }^{1}$, Masatoshi Jinnin ${ }^{2}$ \\ ${ }^{1}$ Department of Plastic Surgery, Wakayama Medical University, Wakayama, Japan; \\ ${ }^{2}$ Department of Dermatology, Wakayama Medical University, Wakayama, Japan; \\ ${ }^{3}$ Department of Diagnostic Pathology, Wakayama Medical University, Wakayama, Japan.
}

SUMMARY In the present study, to identify the clinical significance of the cytokeratin (CK) 20 staining pattern in Merkel cell carcinoma (MCC), we retrospectively analyzed the major clinicopathological and immunohistochemical characteristics of 12 cases of MCC. Typical dot-like pattern was seen in eight of our patients, while four patients showed peripheral staining pattern. Interestingly, all cases of MCC with dot-like CK20 tumor cells occurred in the head and neck region, while those with peripheral CK20 pattern tended to be located in other lesions (forearm, knee, or buttock): The difference of frequency in the head and neck regions was statistically significant. Dot-like CK20 staining pattern may therefore be resulted from ultraviolet exposure. Additionally, although without significance, metastasis was more frequent in those with dot-like CK20 than in peripheral CK20 staining: All patients with peripheral CK20 pattern had complete remission by surgical excision with or without radiation therapy. CK20 staining pattern may be a novel predictor of prognosis.

Keywords Immunohistochemistry, skin, CK20

\section{Introduction}

Merkel cell carcinoma (MCC) is a rare and aggressive epithelial cutaneous neoplasm with neuroendocrine differentiation. It typically appears as solitary redviolaceous tumors in sun-exposed head and neck lesions of elderly people (1); it grows rapidly without pain. Males are more susceptible, and the mean age of patients is reported to be 70 years (2). Merkel cell polyomavirus is detected in $80 \%$ of the lesions. Histopathologically, the tumor typically consists of a dense population of small, round, and uniform cells in the dermis and subcutaneous tissue without change to the overlying epidermis. Differential diagnosis includes the "small round blue cell" tumor group, e.g., metastatic small cell carcinoma of the lung. Immunohistochemical analysis of cytokeratin (CK)20, epithelial membrane antigen, neuron-specific enolase, synaptophysin, chromogranin A, thyroid transcription factor (TTF)-1, S100 protein, leucocyte common antigen, and CD56 has been shown to be useful for the diagnosis (1).

CK20 staining of tumor cells shows perinuclear dot-like patterns in the majority of patients with MCC. Meanwhile, we recently noticed peripheral CK20 staining pattern in a patient subset. In this study, to identify the clinical significance of CK20 staining pattern, we retrospectively analyzed the major clinicopathological and immunohistochemical characteristics of patients with MCC.

\section{Materials and Methods}

\subsection{Clinical assessment and patient material}

The present study includes 12 patients with MCC (6 men and 6 women) who visited Wakayama Medical University Hospital between 2010 and 2020. All patients were diagnosed based on clinical manifestation and immunohistopathological findings. Patient data (age, gender, location, diameter of MCC, immunohistological findings, and prognosis) were collected retrospectively (Table 1).

\subsection{CK20 immunoreactivity}

Skin samples were paraffin-embedded, and sections were dewaxed in xylene and rehydrated in graded alcohols. Hematoxylin and eosin staining was performed as described previously (3).

For immunostaining, anti-cytokeratin 20 rabbit 
monoclonal primary antibody (Roche Diagnostics, Indiana, USA) was optimized for use with VENTANA OptiView DAB IHC Detection Kit (Ventana medical system, Arizona, USA) on automated VENTANA BenchMark ULTRA platform (Roche Diagnostics). CK20-positive cells were randomly observed in five different 800-fold magnification fields under the inverted microscope. Dominant staining patterns were recorded.

\subsection{Statistical analysis}

Statistical analysis was carried out with Fisher's exact test to compare frequency. $P$ values $<0.05$ were considered significant.

\section{Results and Discussion}

3.1. Clinical and histopathological features of MCC patients

We collected the clinical data of 12 patients with MCC for the present study. Detail of one of the patients (case No.3) was previously published as case report focusing on reconstruction surgery (4).

As shown in Table 1, the clinical characteristics of 12 lesions were as follows: mean age was 82.1 years (age range: $75-89$ years), and male:female ratio was 6:6. Distribution of the tumors was 3 lesions in the cheeks, 2 in the eyelids, 1 in the external canthus, 3 in the earlobes, 1 in the forearm, 1 in the lower leg, and 1 in the buttock. The mean diameter of the tumors was 2.6 $\mathrm{cm}$ (range: $1-5 \mathrm{~cm}$ ). Regarding the immunoreactivity of the tumor cells, staining of CK20 was positive in all cases. CD56 was also positive in all seven tested cases. Positive staining of synaptophysin or chromogranin A, the most common neuroendocrine markers, was also observed in eight out of the nine tested cases. None of the cases tested were positive for CK7 or TTF-1 (0/7 and $0 / 9$, respectively). Merkel cell polyomavirus was detected in three cases.

These clinical and immunohistopathological features are generally consistent with previous notions (2). We noticed, however, that there are two different CK20 patterns of tumor cell immunoreactivity: typical dot-like pattern, a perinuclear globular aggregation, was seen in eight cases, while four cases showed peripheral staining patterns. Representative clinical and histopathological findings of cases with dot-like pattern and those with peripheral pattern are shown in Figures 1 and 2, respectively.

\subsection{Clinical significance of different CK20 immunoreactivity of tumor cells}

We then attempted to identify the clinical significance of different CK20 immunoreactivity of tumor cells. There

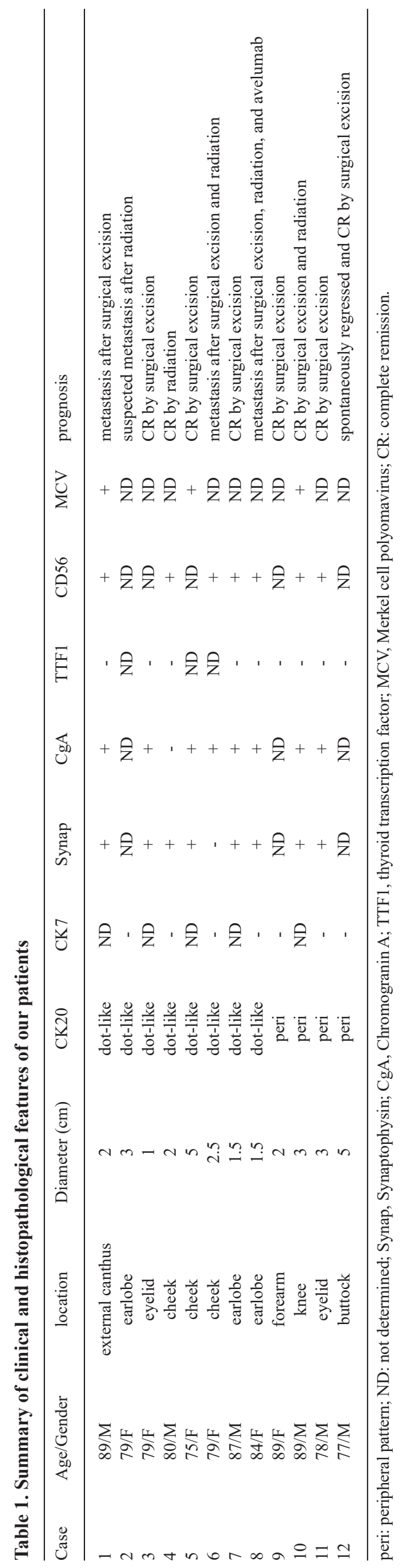




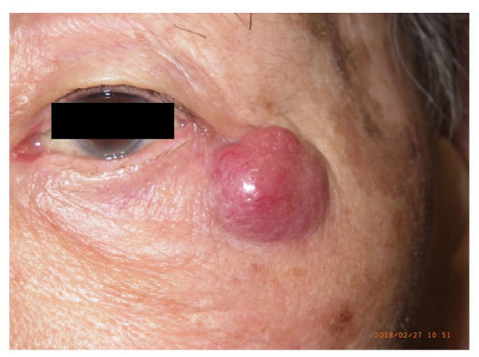

b

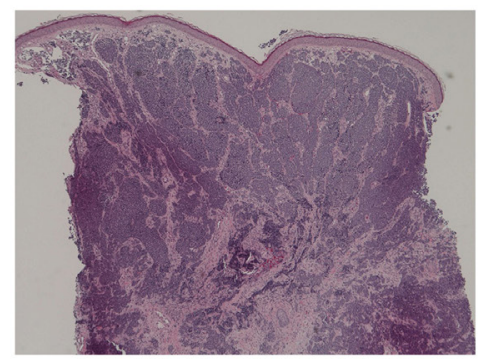

d

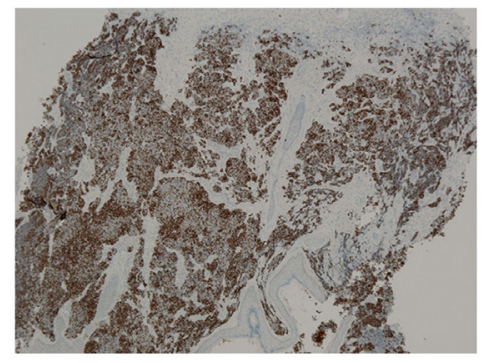

C

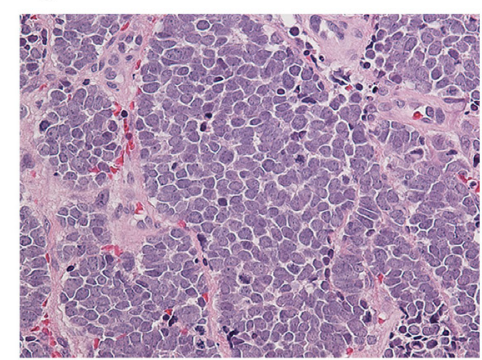

e

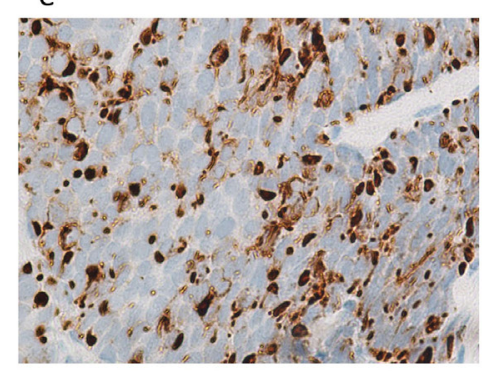

Figure 1. Representative clinical pictures and histopathological findings of a patient with dot-like perinuclear CK20 (patient No.1). (a) Clinical presentation of the tumor on the external canthus. (b) Hematoxylin and eosin staining showing dermal proliferation of small round blue cells (magnification: $\times 40$ ). (c) The tumor cells were round and basophilic with high nuclear:cytoplasmic ratio, hyperchromatic nuclei, and finely stippled salt and pepper chromatin pattern. Mitotic figures were also found (magnification: $\times 400$ ). (d) The tumor cells were positive for CK20 (magnification: $\times 40$ ). (e) CK20 staining showed perinuclear dot-like pattern (magnification: $\times 800$ ).

a

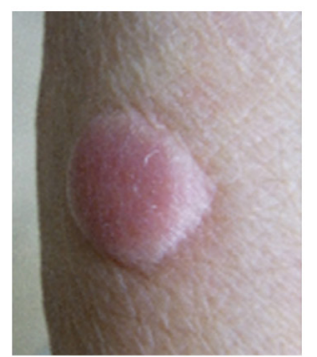

d

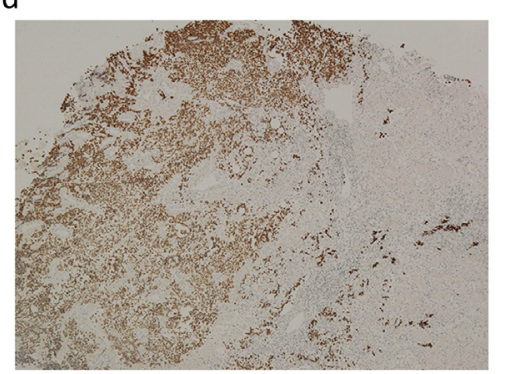

b

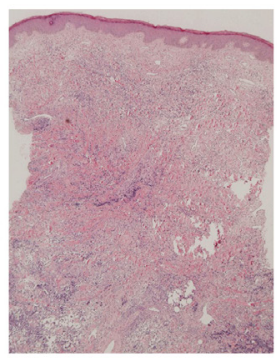

C

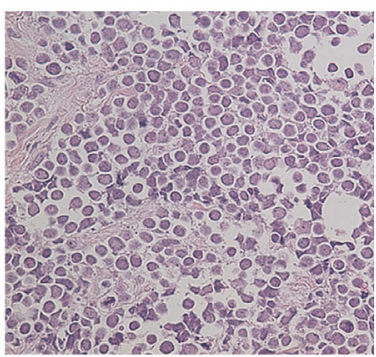

e

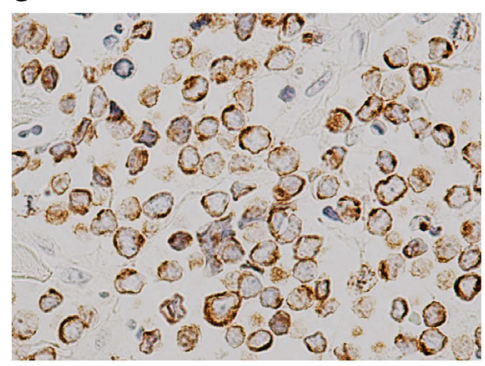

Figure 2. Representative clinical pictures and histopathological findings of a patient with peripheral CK20 (patient No.9). (a) Clinical presentation of a tumor on the forearm. (b) Hematoxylin and eosin staining showing dermal proliferation of small round blue cells (magnification: $\times 40$ ). (c) The tumor cells were round and basophilic with high nuclear:cytoplasmic ratio and finely stippled salt and pepper chromatin pattern (magnification: $\times 400$ ). (d) The tumor cells were positive for CK20 (magnification: $\times 40$ ). (e) CK20 staining showed peripheral nuclear pattern (magnification: $\times 800$ ). was no statistically significant correlation with age, gender, location, or diameter of the tumors. However, all of the MCCs with dot-like CK20 tumor cells occurred in the head and neck region, while those with peripheral CK20 pattern tended to be located in other lesions (forearm, knee, or buttock), and the difference of frequency in head and neck lesions was statistically significant $(100.0 \%$ vs. $25.0 \%, p=0.0182$ by Fisher Exact test).

In addition, although insignificant, metastasis was more frequent in those with dot-like CK20 (50.0\% vs. $0.0 \%, p=0.21$ ) than peripheral CK20 staining; all patients with peripheral CK20 pattern resulted in complete remission by surgical excision with or 
without radiation therapy. There was no difference in the positivity of other immunostaining between the two groups.

CK20 is a type I cytokeratin, which is a major cellular protein of mature enterocytes and goblet cells. CK20 expression is usually therefore observed in normal gastric and intestinal mucosa (5), and CK20 staining is used to identify a range of adenocarcinoma arising from CK20-positive epithelia, including colorectal cancer. In addition, tumor cells of MCC are well known to express CK20, but are absent in metastatic lung cancer.

CK20 staining pattern of enterocytes is usually diffuse, while dot-like perinuclear pattern was characteristic of the tumor cells of $\operatorname{MCC}(6,7)$. To our knowledge, the mechanism of dot-like CK20 staining, however, remains unknown. Furthermore, we noticed peripheral staining pattern of CK20 in a subset of MCC patients. We found that all MCCs with dot-like CK20 tumor cells occurred in the head and neck region, while those with peripheral pattern CK20 tended to be located in the trunk and extremities, and the difference was statistically significant. Established risk factors for MCC are immunosuppression and extensive sun exposure: increased frequency of MCCs in patients with acquired immune deficiency syndrome and in those receiving immunosuppressive therapy has been reported (7). The incidence rate of cutaneous MCC is also increased by sun exposure (7). Here, all MCCs with dot-like CK20 tumor cells occurred in the head and neck region, so dot-like CK20 staining pattern may be the result of ultraviolet exposure. There has been quite a few report to focus on the staining pattern of CK20 in patients with MCC.

Metastasis was more frequent in patients with dotlike CK20 than in peripheral CK20 staining, whereas all patients with peripheral CK20 pattern resulted in complete remission by surgical excision with or without radiation. The rates of local and regional recurrence were reported to be $29 \%$ and $59 \%$, respectively, in MCC patients (7). In addition, the estimated mortality rate for all patients with MCC is between 25\% and 35\% (8). Previous studies reported poor prognostic factors to be male sex, tumor size $>5 \mathrm{~mm}$ or $>20 \mathrm{~mm}$, location (in the buttock/thigh/trunk or in the head), advanced clinical stage, small cell size, high mitotic index, diffuse growth pattern, as well as p53 positivity (9). CK20 staining pattern may also be a novel predictor of prognosis in MCC. Detailed research with a larger number of samples is necessary to prove clinical significance of CK20 staining pattern.

\section{Acknowledgements}

We acknowledge proofreading and editing by Benjamin
Phillis at the Clinical Study Support Center, Wakayama Medical University.

\section{Funding: None.}

Conflict of Interest: The authors have no conflicts of interest to disclose.

\section{References}

1. Llombart B, Monteagudo C, Lopez-Guerrero JA, Carda C, Jorda E, Sanmartin O, Almenar S, Molina I, Martin JM, Llombart-Bosch A. Clinicopathological and immunohistochemical analysis of 20 cases of Merkel cell carcinoma in search of prognostic markers. Histopathology. 2005; 46:622-634.

2. Brissett AE, Olsen KD, Kasperbauer JL, Lewis JE, Goellner JR, Spotts BE, Weaver AL, Strome SE. Merkel cell carcinoma of the head and neck: a retrospective case series. Head Neck. 2002; 24:982-988.

3. Tanaka C, Fujimoto M, Hamaguchi Y, Sato S, Takehara $\mathrm{K}$, Hasegawa M. Inducible costimulator ligand regulates bleomycin-induced lung and skin fibrosis in a mouse model independently of the inducible costimulator/ inducible costimulator ligand pathway. Arthritis Rheum. 2010; 62:1723-1732.

4. Sakata Y, Okuda K, Wada Y, Kumegawa S, Kusuhara $\mathrm{H}$, Isogai $\mathrm{N}$, Asamura $\mathrm{S}$. Eye comfort and physiological reconstruction of an entire upper eyelid defect. Eplasty. 2020; 20:e5.

5. Moll R, Schiller DL, Franke WW. Identification of protein IT of the intestinal cytoskeleton as a novel type I cytokeratin with unusual properties and expression patterns. J Cell Biol. 1990; 111:567-580.

6. Jovanovic I, Tzardi M, Mouzas IA, Micev M, Pesko P, Milosavljevic T, Zois M, Sganzos M, Delides G, Kanavaros P. Changing pattern of cytokeratin 7 and 20 expression from normal epithelium to intestinal metaplasia of the gastric mucosa and gastroesophageal junction. Histol Histopathol. 2002; 17:445-454.

7. Shnayder Y, Weed DT, Arnold DJ, Gomez-Fernandez C, Bared A, Goodwin WJ, Civantos FJ. Management of the neck in Merkel cell carcinoma of the head and neck: University of Miami experience. Head Neck. 2008; 30:1559-1565

8. Herbert HM, Sun MT, Selva D, et al. Merkel cell carcinoma of the eyelid: management and prognosis. JAMA Ophthalmol. 2014; 132:197-204.

9. Carson HJ, Reddy V, Taxy JB. Proliferation markers and prognosis in Merkel cell carcinoma. J Cutan Pathol. 1998; 25:16-19.

Received May 18, 2021; Revised June 25, 2021; Accepted June 27, 2021.

*Address correspondence to:

Yutaka Inaba, Department of Dermatology, Wakayama Medical University, 811-1 Kimiidera, Wakayama, Wakayama, 641-0012, Japan.

E-mail: ptfjk298@wakayama-med.ac.jp 